Revue des patrimoines

\title{
Pour une connaissance partagée du patrimoine rural
}

Pascal Liévaux

\section{Q OpenEdition \\ 1 Journals}

\section{Édition électronique}

URL : http://journals.openedition.org/insitu/2295

DOI : 10.4000/insitu.2295

ISSN : 1630-7305

Éditeur

Ministère de la Culture

\section{Référence électronique}

Pascal Liévaux, «Pour une connaissance partagée du patrimoine rural », In Situ [En ligne], 5 | 2004, mis en ligne le 06 novembre 2012, consulté le 21 septembre 2020. URL : http://journals.openedition.org/ insitu/2295; DOI : https://doi.org/10.4000/insitu.2295

Ce document a été généré automatiquement le 21 septembre 2020.

\section{(c) (i) (9)}

In Situ Revues des patrimoines est mis à disposition selon les termes de la licence Creative Commons Attribution - Pas d'Utilisation Commerciale - Pas de Modification 4.0 International. 


\title{
Pour une connaissance partagée du patrimoine rural
}

\author{
Pascal Liévaux
}

Figure 1

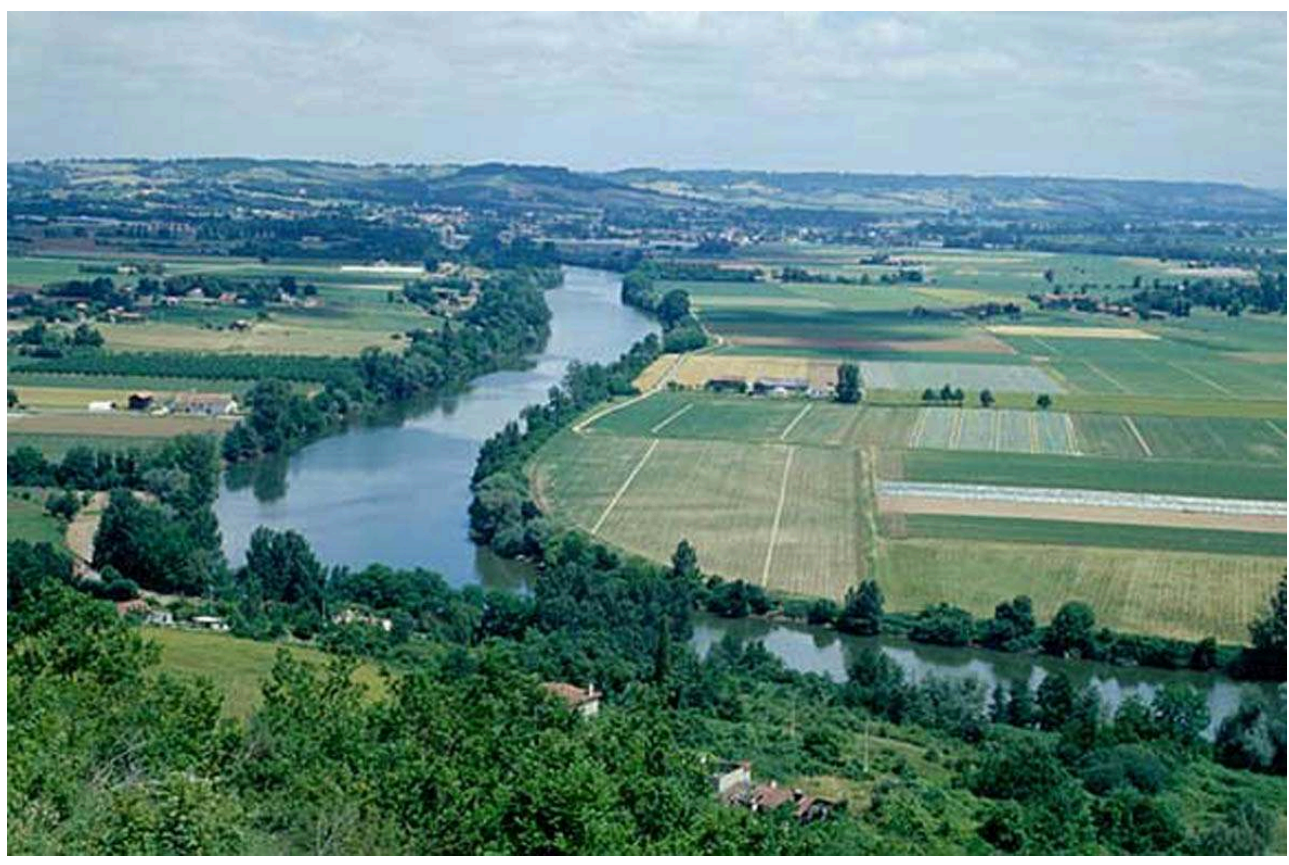

Bourran, Lot-et-Garonne. Vue de la plaine du Lot depuis le sommet du Pech de Berre vers le nord-est Phot. Inv. M. Dubau @ Inventaire général, ADAGP, 1998

1 Ce cinquième opus de la revue In Situ et un numéro prochain rassemblent des textes consacrés au patrimoine rural français, domaine auquel les services régionaux de l'Inventaire général ont consacré de nombreuses études ${ }^{1}$ dont témoignent une documentation $^{2}$ et une bibliographie ${ }^{3}$ considérables (fig. $\mathbf{n}^{\circ} \mathbf{1}$ ). 
2 Menant leurs travaux au sein de l'Inventaire, des Monuments Historiques ou de l'Université, leurs auteurs y font le point sur des travaux en cours, tirent les conclusions d'opérations abouties ou présentent des méthodes d'approche du terrain aussi bien que des sources archivistiques susceptibles d'ouvrir de nouvelles pistes à la recherche et d'enrichir la connaissance du territoire rural au plan national.

Figure 2

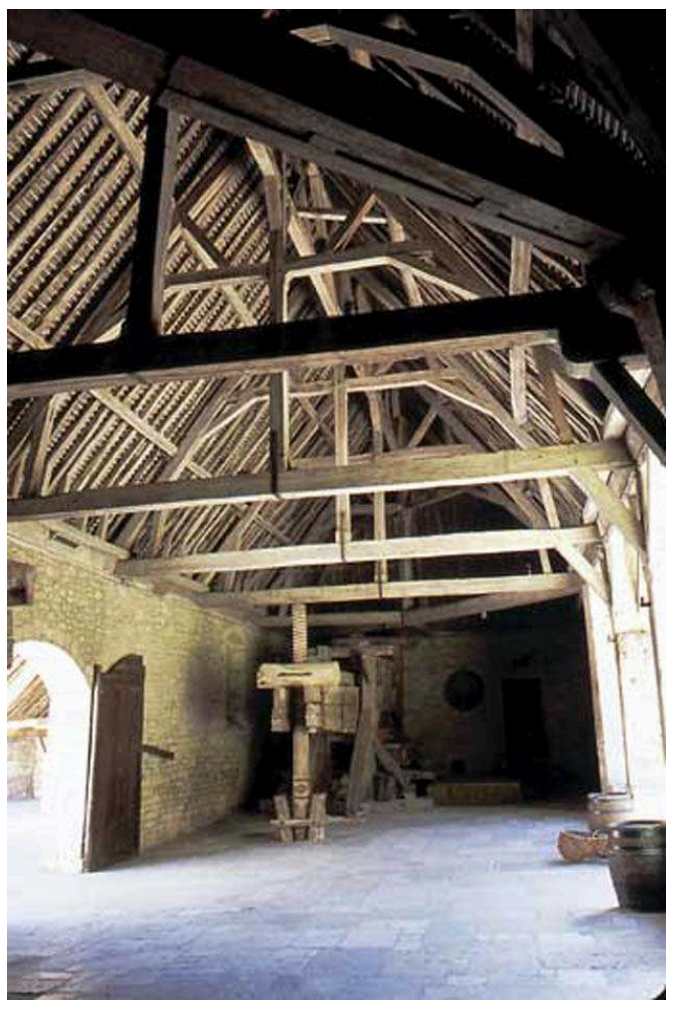

Le Clos-Vougeot, Côte-d'Or. Cuverie du château, charpente et pressoir Phot. Inv. M. Thierry @ Inventaire général, ADAGP, 2003

3 Sur ce dernier point, le travail de l'Inventaire général fut longtemps précurseur en ce qu'il propose une vision globale du patrimoine sur son territoire naturel, qui fait la part belle à un bâti, et à des objets délaissés par l'histoire de l'art traditionnelle, tels que fermes, granges, hâloirs à fromage ou pressoirs (fig. $\mathbf{n}^{\circ} 2$ ), longtemps absents des listes d'églises et de châteaux remarquables qui constituèrent jusqu'au milieu du siècle dernier l'essentiel des politiques de conservation ${ }^{4}$.

Il est donc important de disposer d'un socle de connaissances procédant d'une approche historique et globale du territoire, qui ne dissocie pas artificiellement les

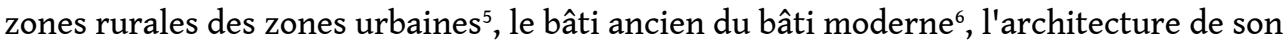
environnement naturel, l'abbaye ou la grande demeure des parcs, des fermes, des granges, des moulins et des ouvrages d'art qui en dépendaient, ${ }^{7}$ mais les étudie en réseaux, qui ne distingue pas le patrimoine reconnu des châteaux ${ }^{8}$ et des abbayes d'un " petit patrimoine » qui serait celui des fermes, des cabanons méridionaux, des cases créoles $^{9}$ et du mobilier qu'ils abritent.

5 L'élargissement de la notion de patrimoine ${ }^{10}$, à laquelle l'Inventaire général apporta sa pierre, et celle, concomitante, du champ opérationnel de la protection, contribuèrent sans aucun doute à infléchir la tendance ${ }^{11}$ mais, il faut bien le constater, à l'heure où le 
territoire français connaît des bouleversements majeurs résultant de la transformation des modes de vie et de la multiplication d'aménagements aux conséquences paysagères de plus en plus lourdes, les réflexions menées ici et là n'ont pas encore permis, en dépit de l'urgence qu'il y aurait à le faire, de fixer les principes d'une action véritablement concertée pour la préservation des patrimoines naturel et culturel, autour de laquelle se rassembleraient tous les acteurs patrimoniaux.

C'est cette approche aux échelles territoriales et aux questionnements multiples, (techniques, typologiques, stylistiques, ethnologiques, archéologiques...), envisageant la permanence et l'évolution des structures agraires, des formes architecturales et des outils de production ${ }^{12}$, qu'illustrent les articles rassemblés dans ce numéro organisé en trois parties traitant successivement des sources (fig. $\mathbf{n}^{\circ} \mathbf{3}$ ), de l'assiette territoriale et des fonctions du patrimoine rural.

Figure 3

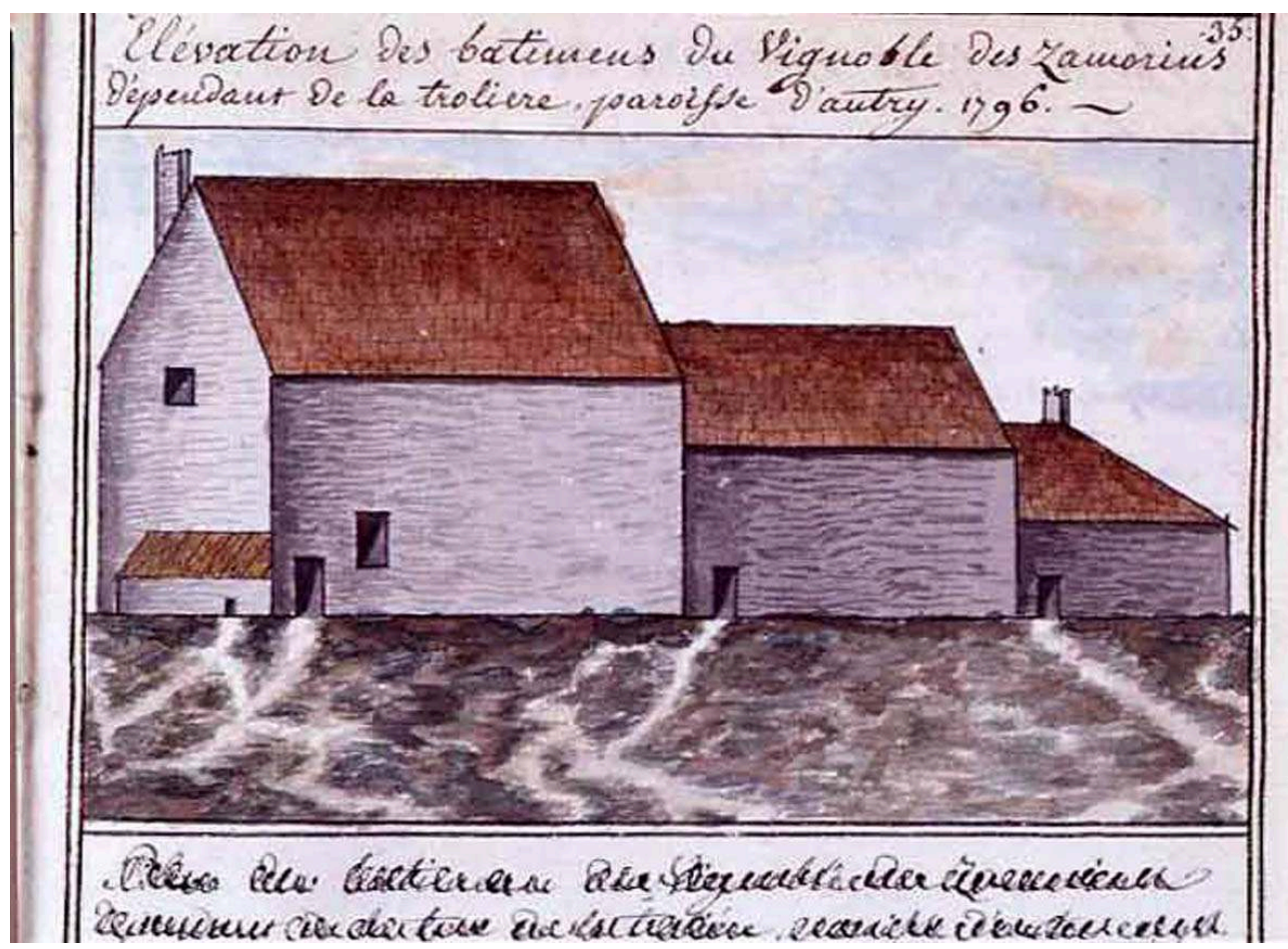

Saint-Menoux, Allier. Domaine de la Tardivonnerie, plan à terre, dessin à la plume aquarellé, 1784-1789 (A. D. Allier, 1 J 245)

Phot. Inv. R. Choplain et R. Maston ( Inventaire général, ADAGP, 2003

7 On verra dans la première partie que parallèlement aux vestiges physiques, les sources archivistiques apportent non seulement des informations précieuses sur le bâti, la nature des cultures et l'implantation des villages, y compris parfois pour les périodes les plus reculées ${ }^{13}$, mais permettent aussi d'élaborer de nouvelles méthodes d'enquête ${ }^{14}$.

La seconde partie, essentiellement consacrée aux résultats de travaux portant sur de vastes territoires ${ }^{15}$, tente notamment d'appréhender dans toute sa complexité la question des types architecturaux et de leur diffusion, de leur permanente évolution, de leur étonnante capacité d'adaptation aux conditions physiques, climatiques, historiques, économiques et sociales les plus diverses. 
9 La troisième partie complète cette approche typologique par l'étude d'architectures et d'objets, outils de production, conçus pour la réalisation de produits aussi élaborés que les fromages du pays d'Auge ${ }^{16}$ ou les vins de Bourgogne ${ }^{17}$, dans une parfaite adéquation de la forme et de la technique au travail des hommes.

10 Autant d'apports à l'histoire, forcément pluridisciplinaire, du monde rural, qui contribueront certainement à la préservation éclairée, dynamique et durable de son patrimoine.

\section{NOTES}

1. Soumis au crible d'une méthode d'investigation constamment perfectionnée, les territoires explorés par les chercheurs des vingt-deux régions métropolitaines et des DOM-TOM font l'objet de dossiers riches de présentations générales qui permettent de saisir les caractéristiques physiques, historiques et patrimoniales des différentes aires d'étude, mettant en perspective les monographies d'objets et d'édifices sélectionnés pour leur caractère exceptionnel ou, a contrario, représentatif.

2. Le travail mené à l'Inventaire général sur le patrimoine rural depuis quatre décennies peut être consulté pour partie sur les bases de données Mérimée, Palissy et Mémoire et, dans son intégralité, auprès des Services régionaux.

3. Voir les collections nationales des Cahiers, Images et Itinéraires du Patrimoine.

4. Voir Poirrier, Philippe et Vadelorge, Loïc (dir.). Pour une histoire des politiques du patrimoine. Paris : Ministère de la Culture ; Comité d'histoire, 2003.

5. De plus en plus floue, la frontière entre ville et campagne n'a jamais été vraiment pertinente au regard de l'histoire. Plusieurs travaux récents abordent ce sujet auquel la revue Histoire urbaine a consacré un récent numéro: Urbanité et ruralité. Histoire urbaine, Paris, $\mathrm{n}^{\circ} 8$, décembre 2003.

6. Les bornes chronologiques des opérations d'inventaire sont fixées à l'an 400 pour la limite haute, à 30 ans avant la réalisation de l'enquête pour la limite basse.

7. Voir notamment les articles de Laurent Ursulet: L'Habitation Beauséjour : grandeur et pérennité d'une plantation martiniquaise et de Franck Tournadre : De l'aile conventuelle à destination des convers au bâtiment agricole à fonction multiple: le cas des abbayes cisterciennes de Chaloché (Maine-et-Loire), Preuilly (Seine-et-Marne) et Valence (Vienne).

8. Voir l'article de Frédérique Guilbaud: Une demeure aux champs de la Renaissance : le manoir tourangeau de La Courtinière à Beaumont-en-Véron.

9. Voir l'article de Christophe Denise : Une histoire évolutive de l'habitat martiniquais et de Marie-Pascale Mallé : Les maisons des Noirs marrons de Guyane.

10. La bibliographie sur ce sujet est d'une extrême richesse, on connaît l'ouvrage fondateur de Pierre Nora (NORA, Pierre (dir.). Les lieux de mémoire. Paris: Gallimard, 3 vol., 1984-86. Sur l'évolution du concept de monument, voir l'article stimulant de Sandro Scarrocchia : Scarrocchia, Sandro. Le concept moderne de monument et la valeur de l'ancien. Revue de l'Art, Paris, $n^{\circ} 145 / 2004-3$, p. 19-28.

11. Par la mise en place de secteurs sauvegardés (loi dite " Malraux » du 4 août 1962) et de zones de protection du patrimoine urbain et paysager, dites ZPPAUP (loi du 7 janvier 1983). Ces outils de protection furent conçus avant tout pour les espaces urbains. Les ZPPAUP, de plus en plus 
nombreuses en territoire rural, ne permettent cependant pas de répondre à toutes les attentes en matière de protection du patrimoine rural.

12. Voir les articles d'Hubert Maheux: Champs ouverts, habitudes communautaires et villages en alignements dans le nord de la Loire-Atlantique : des micro-sociétés fossilisées dans l'Ouest bocager et de Christophe Charlery : Maisons de maître et habitations coloniales dans les anciens territoires français de l'Amérique tropicale.

13. Voir à ce propos l'article de Marie-Elisabeth Bruel: L'apport des archives à l'étude du patrimoine rural : l'exemple des communes d'Autry-Issards et de Saint-Menoux (canton de Souvigny, Allier).

14. Voir l'article de Christine Toulier : L'apport du cadastre à la recherche sur les jardins historiques : l'exemple du département du Maine-et-Loire.

15. Voir plus particulièrement les articles d'Hélène Mousset : L'habitat rural des vallées de la Baïse et du Lot : contexte agraire et paysager, de Marie-Sylvie Grandjouan : Le patrimoine rural en Languedoc-Roussillon: acquis et perspectives du travail d'inventaire et de Christophe Charlery: Maisons de maître et habitations coloniales dans les anciens territoires français de l'Amérique tropicale.

16. Voir l'article de Yannick Lecherbonnier : Architectures fromagères en pays d'Auge.

17. Voir l'article de Bernard Lauvergeon : Les grands pressoirs bourguignons pré-industriels : essai de chrono-typologie.

\section{AUTEUR}

\section{PASCAL LIÉVAUX}

Conservateur du patrimoine, département recherche, méthodes et expertise, sous-direction de l'archéologie, de l'ethnologie et de l'inventaire, direction de l'architecture et du patrimoine. pascal.lievaux@culture.gouv.fr 